\title{
Some non-linear parametric resonance oscillations of dumbell satellite in elliptical orbit
}

\author{
A. Narayan and M. D. Pandey* \\ Department of Mathematics, Bhilai Institute of Technology, Bhilai House Durg, India \\ *Corresponding author E-mail: mithileshpandey_1978@yahoo.co.in
}

\begin{abstract}
Some non-linear parametric resonance oscillations of Dumbell satellite in elliptical orbit in the central gravitational field of the Earth under the combined influence of Earth magnetic field ,oblateness of the Earth and some external periodic forces of general in nature has been studied.The system comprises of two charged material particles connected by a light flexible and inextensible cable ,moves with taut cable like a dumbell satellite around the Earth in elliptical orbit.The central gravitational field of the Earth is the main force governing the motion of the system and various perturbing forces influencing the system are disturbing in nature. Non-linear oscillations of dumbbell satellite about the equilibrium position in the neighbourhood of the parametric resonance $\omega=\frac{1}{2}$, has been investigated, exploiting the well known asymptotic method due to Bogoliubov,Krilov and Metropoloskey, considering 'e' to be a small
\end{abstract} parameter. The analysis of stability of the system has been discussed due to Poincare method.

Keywords: Non-linear oscillations, Dynamical system, Perturbing forces, Cable connected satellite.

\section{Introduction}

The present paper deals with non-linear parametric resonance oscillations of cable connected satellite system connected by a light flexible and inextensible cable in the gravitational field of the Earth.The spacecraft studied in the present research works is also subject to the influence of the three different perturbations (i) the small eccentricity of the elliptical orbit (ii)a magnetic field of the Earth it is due to the interaction between the Earth's magnetic field and the magnetic moment of the spacecraft (iii) the oblateness of the Earth and (iv) the small external periodic forces of the general in nature.The satellites which are connected by non conducting cable are considered to be a charged material particles and the motion of one of the particles is studied relative to their centre of mass, under the assumption that later moves along the elliptical orbit.The cable connecting the two satellites is considered to be taut and non elastic in nature such that the system moves like a dumbell satellite.Many space configurations have been proposed and investigated, the stability of the system in elliptical orbit under the various perturbing forces. The simplest space configuration is dumbell satellite system, when the two satellites are connected by a rod (4), two or more satellites are connected by a tether Krupa etal (6,7), Beletsky \& Levin (2), Mishra \&Modi(9), and the two satellites are connected by spring (13). All these authors have mentioned numerous important applications of the system and their stability of relative equilibrium of the system moves in a circular and elliptical orbit .Beletsky (1), Beletsky\& Novikova (3) studied the motion of a system of two satellites connected by a light,flexible and inextensible string in the central gravitational field of force relative to their centre of mass of the system, when the centre of mass is itself assume to move along a Keplerian elliptical orbit ,under the assumption that the two satellites are moving in the plane of motion of centre of mass. Singh (16, 17), dealt the some problem in its general form. He further investigated by considering the analysis of relative motion of the system for the elliptical orbit of the centre of mass in two dimensional as well as three dimensional cases. Narayan \&Singh $(10,11,12)$, studied non-linear oscillations due to the solar radiation pressure provided the centre of mass of the system moves along an elliptical orbit. Singh etal $(18,19)$, studied the non-linear effects of the Earth's oblateness in the motion and stability of cable connected satellites system in elliptical orbit. Das etal (5), and Narayan etal (23), studied the non-linear effects of Earth's magnetic field in the stability of cable connected system in inclined and equatorial orbit The present paper is devoted to the analysis of the combined effects of the magnetic field of the Earth, Oblateness of the Earth and the external periodic forces of general nature on non-linear parametric resonance oscillations of cable connected satellites system in elliptical orbit. The perturbing forces due to the Earth's magnetic field results from the 
interaction between spacecraft's residual magnetic field and the geomagnetic field. The perturbing force is arising due to magnetic moments, eddy current and hysteresis, out of these the spacecraft magnetic moment is usually the dominant sources of disturbing effects. Nevertheless a distant satellite beyond gravitational field of the Earth, in addition to above mentioned forces(non uniform gravitational field and magnetic field of the Earth ), it could still expected to be affected by general nature of external forces could arise due to dissipation of energy generated on account of friction of bodies in the atmosphere by tidal forces gravitational radiation, these forces though small can significantly affect the oscillations of the system under considerations. These forces could be modeled as frictional forces with small dissipation coefficient. Furthermore, the forces generated by the multipole moments and absorption of gravitational waves at resonance frequency could be characterized as external periodic forces having a slowly varying frequency and these forces could be estimated by certain model assumption (20).

Thus, in order to study non-linear parametric resonance oscillations of dumbell satellite in elliptical orbit on realistic basis, it is essential to consider the combined influences of the Earth's magnetic field, oblateness of the Earth and the periodic force of general nature.

\section{Equation of motion}

The combined influence of the geomagnetic field and oblateness of the Earth and external periodic forces of general nature on the motion and stability of a cable connected satellites system in the central gravitational field of Earth in elliptical orbit has been considered.

The equation of motion of satellite in the central gravitational field of Earth under the combined influence of Earth's magnetic field, oblateness of the Earth is given by:

$$
\begin{aligned}
(1+e \cos \mathrm{v}) \psi^{\prime \prime}-2 e & \psi^{\prime} \sin v+3 \sin \psi \cdot \cos \psi+5 A(1+e \cos v)^{2} \sin \psi \cdot \cos \psi \\
= & B \cos \delta(1+e \cos \mathrm{v}) \sin \psi-B \cos \delta \cdot \sin \mathrm{v} \cdot \cos \psi+2 e \sin \mathrm{v}
\end{aligned}
$$

The non-linear oscillations described by (2.1), take place as long as inequality given below is satisfies

$$
\begin{aligned}
& (1+e \cos \mathrm{v})^{4}\left(\psi^{\prime}+1\right)^{2}+(1+e \cos \mathrm{v})^{3}\left(3 \cos ^{2} \psi-1\right) \\
& \quad-B \cos \delta(1+e \cos \mathrm{v})^{3}\left[\cos \psi+e \cos (\psi+\mathrm{v})-A(1+e \cos \mathrm{v})^{3}\left(4 \cos ^{2} \psi-\sin ^{2} \psi\right)\right] \geq 0
\end{aligned}
$$

where $\mathrm{v}$ and $\mathrm{e}$ are respectively true anomaly and eccentricity of the orbit of centre of mass of the system. The prime denotes differentiation with respect to true anomaly $\mathrm{v}$, where $\psi$ is the angular derivative of the line joining the centre of mass of the cable connecting the satellite with the stable position of equilibrium. A is the oblateness due to the Earth and $\mathrm{B}$ is the magnetic field of the Earth. The equation (2.1), represents the oscillations of the system about the stable position of equilibrium in which the system lies wholly along the radius vector joining the centre of mass and the centre of force Narayan et al (2010). Substituting $2 \psi=\eta$ in (2.1), the equation of motion can be expressed as follows:

$$
\begin{aligned}
& \eta^{\prime \prime}+3 \sin \eta=4 e \sin \mathrm{v}+e \eta^{\prime} \sin \mathrm{v}-5 \mathrm{~A}(1+\mathrm{ecos} \mathrm{v})^{2} \sin \eta-\eta^{\prime \prime} e \cos \mathrm{v} \\
& +2 B \cos \delta \sin \frac{\eta}{2}+2 e B \cos \delta \sin \left(\eta-\frac{v}{2}\right)+E \sin v \mathrm{v}+\gamma \eta^{\prime}
\end{aligned}
$$

Equation (2.3), describes non-linear oscillations of the dumbell satellite in elliptical orbit, in central gravitational field of the Earth's along with the magnetic field of the Earth, Oblateness of the Earth , friction force and periodic force, where $\gamma$ and $E$ are some phenomenological parameter characterizing the tidal and periodic forces acting on the system and they are assumed to be the order of ' $e$ '. Where $v$ is the frequency of the external periodic force. However, these parameters can be determined from specific model assumptions concerning these problems. 


\section{Non-linear parametric resonance oscillations of dumbell satellite system about the position of equilibrium for small eccentricity}

The non-linear oscillations of the dumbbell satellite under the influence of the above mentioned forces described by (2.3), will be investigated for the resonance case, on the assumption that $\gamma$ and $E$ are of order ' $\mathrm{e}$ '. This is justified on account of the fact that these estimates are always concerned with a certain model assumption. Hence for our conveinence, setting $E=e E_{1}, \lambda=e \lambda_{1}$ and $B \cos \delta=e B \cos \delta$, the equation (2.3), is expressed in definite form which is suitable for our further analysis:

$$
\begin{aligned}
\eta^{\prime \prime}+\omega^{2} \eta & =e\left[\beta(\eta-\sin \eta)+2 \eta^{\prime} \sin v+4 \sin v-\eta^{\prime \prime} \cos v\right. \\
& \left.+E_{1} \sin v v+\gamma^{\prime} \eta^{\prime}+2 B \cos \delta \sin \frac{\eta}{2}-5 A \sin \eta\right] \\
& +e^{2}\left[10 A \cos v \sin \eta+2 B \cos \delta \sin \left(v-\frac{\eta}{2}\right)\right]
\end{aligned}
$$

where $\omega^{2}=3$ and $\beta=\left(\frac{\omega^{2}}{e}\right)$. More over the non-linearity term $(\eta-\sin \eta)$ will be assumed to be the order of $e$.

The system described by equation (3.1), moves under the forced vibration due to the presence of the magnetic field of the Earth, oblateness of the Earth and the external periodic forces of general nature on the right hand side of the equation. These periodic sine forces are of perturbative nature as long as the period of oscillations of the system is different from the period of sine force for which solution is obtain (25). As the period of sine force is always changing, it may become equal to the sine force in that case the periodic sine force plays vital role in the oscillatory motion of the system. While examining the non resonance case, Narayan and M.D.Pandey (31), they conclude that the system experience parametric resonance behavior at and near $\omega=\frac{1}{2}$; where the non-resonance solution fails. We are benefitted by the smallness of the eccentricity ' $\mathrm{e}$ ' in equation (3.1), hence the solution of the differential equation may be obtained by exploiting the Bogoliubov, Krilov and Metropoloskey method (21).

We construct the asymptotic solutions of the system representing (3.1), in the most general case , which is valid at and near the parametric resonance $\omega=\frac{1}{2}$, exploiting the well known Bogoliubov, Krilov and Metropoloskey method (21).The solution of equation (3.1), in the first approximation will be sought in the form:

$$
\begin{aligned}
& \eta=a \cos \left(\frac{v}{2}+\theta\right) \\
& \frac{d a}{d \mathrm{v}}=e A_{1}(a, \theta) \\
& \frac{d \theta}{d \mathrm{v}}=\left(\omega-\frac{1}{2}\right)+e B_{1}(a, \theta) .
\end{aligned}
$$

where $A_{1}(a, \theta)$ and $B_{1}(a, \theta)$ are particular solution periodic with respect to ' $\theta$ ' of the system.

$$
\begin{aligned}
& \left(\omega-\frac{1}{2}\right) \frac{\partial A_{1}}{\partial \theta}-2 a \omega B_{1}=\frac{1}{2 \pi^{2}} \sum_{\sigma=-\infty}^{\sigma=+\infty} e^{-2 i \sigma \theta} \int_{0}^{2 \pi} \int_{-0}^{2 \pi} f\left(a, \eta, \eta^{\prime}, \eta^{\prime \prime}\right) e^{-2 i \sigma \theta^{\prime}} \cos k d \mathrm{v} d k \\
& a\left(\omega-\frac{1}{2}\right) \frac{\partial B_{1}}{\partial \theta}+2 \omega A_{1}=-\frac{1}{2 \pi^{2}} \sum_{\sigma=-\infty}^{\sigma=+\infty} e^{-2 i \sigma \theta} \int_{0}^{2 \pi} \int_{-0}^{2 \pi} f\left(a, \eta, \eta^{\prime}, \eta^{\prime \prime}\right) e^{-2 i \sigma \theta^{\prime}} \sin k d \mathrm{v} d k
\end{aligned}
$$

where $2 \theta=2 k-\mathrm{v}=2 \theta^{\prime}$ and $f\left(a, \eta, \eta^{\prime}, \eta^{\prime \prime}\right)$ is the coefficient of ' $\mathrm{e}$ ' on the right hand side of equation (3.1).

Simple integration of $f\left(a, \eta, \eta^{\prime}, \eta^{\prime \prime}\right)$ gives us: 


$$
\begin{aligned}
& \left(\omega-\frac{1}{2}\right) \frac{\partial A_{1}}{\partial \theta}-2 a \omega B_{1}=e\left[\beta\left(a-2 J_{1}(a)\right)-\frac{a}{2} \cos 2 \theta+4 B \cos \delta J_{1}\left(\frac{a}{2}\right)-10 A J_{1}(a)\right] \\
& a\left(\omega-\frac{1}{2}\right) \frac{\partial B_{1}}{\partial \theta}+2 \omega A_{1}=\gamma_{1} a+\frac{a}{2} \sin 2 \theta
\end{aligned}
$$

where $J_{1}(a)$ is the Bessel function of the first order

$$
\begin{aligned}
& \sin (a \cos \theta)=2 \sum_{k=0}^{\infty}(-1)^{k} \cdot J_{2 k+1}(a) \cdot \cos (2 k+1) \theta ; \\
& \cos (a \cos \theta)=J_{0}(a)+2 \sum_{k=0}^{\infty}(-1)^{k} \cdot J_{2 k}(a) \cdot \cos 2 k \theta .
\end{aligned}
$$

where $J_{k}, k=01,2,3 \ldots \ldots$. stands for Bessel's function. The periodic solution of the system given by equations (3.6) can obtained as :

$$
\begin{aligned}
& A_{1}=\left[\frac{\gamma_{1} a}{2 \omega}+\frac{a}{2} \sin 2 \theta\right] \\
& B_{1}=\left\{-\frac{\left(a-2 J_{1}(a)\right.}{2 a \omega}\right\}+\frac{\cos 2 \theta}{2}-\frac{2 B \cos \delta}{a \omega} J_{1}\left(\frac{a}{2}\right)+\frac{5 A J_{1}(a)}{a \omega}
\end{aligned}
$$

where the amplitude ' $a$ ' and phase ' $\theta$ ' are the given by the system of differential equations.

$\frac{d a}{d \mathrm{v}}=\frac{\gamma a}{2 \omega}+\frac{a}{2} \sin 2 \theta$

$\frac{d \theta}{d \mathrm{v}}=\left(\omega-\frac{1}{2}\right)-\frac{1}{2 a \omega}\left[\left\{a-2 J_{1}(a)\right\}\right]+\frac{e \cos 2 \theta}{2}+\frac{5 e A J_{1}(a)}{a \omega}-\frac{2 e B \cos \delta}{a \omega} J_{1}\left(\frac{a}{2}\right)$.

It is clear from (3.9), that there is absence of external periodic force $E \sin v$ v on the amplitude and phase of the oscillatory system in first approximation at this overtone. But the presence of dissipative force and the magnetic field of the Earth introduces a correction in the amplitude of the system. The set of equations (3.9), cannot be integrated in 'a'. closed form due to dependence of right hand side ' $a$ ' and' $\theta$ '. However the qualitative aspects of the solution can be examined with the help of Poincare theory (22).

$\frac{d a}{d \mathrm{v}}=\frac{\gamma a}{2 \omega}+\frac{a e \sin 2 \theta}{2}$.

$\frac{d \theta}{d \mathrm{v}}=\omega_{e}-\frac{1}{2}+\frac{e}{2} \cos 2 \theta$

where $\omega_{e}=\omega-\left(\frac{a^{2}}{16 \omega}+\frac{B a^{2} \cos \delta}{64 \omega}-\frac{5 a^{2} A}{16 \omega}\right)$

The parameter $\omega_{e}(a)$ introduced here the equivalent frequency of non linear oscillations of the dumbbell satellite system when the impressed force is absent. We now examined the stationary regime of oscillation of the system in the first approximation. The stationary state of oscillations is defined by $\frac{d a}{d \mathrm{v}}=0$ and $\frac{d \theta}{d \mathrm{v}}=0$.

Hence from the set of equation (3.9), retaining up to the second order termed in the amplitude, we obtain:

$$
\begin{aligned}
& {\left[\frac{\gamma}{2 \omega}+\frac{e \sin 2 \theta}{2}\right] a=0, a \neq 0 ;} \\
& {\left[\omega_{e}-\frac{1}{2}\right]+\frac{e}{2} \cos 2 \theta=0 .}
\end{aligned}
$$


Eliminating the phase $\theta$ in (3.12), we obtain:

$$
\left[\omega_{e}-\frac{1}{2}\right]^{2}=\left[\frac{e^{2}}{4}-\frac{\gamma^{2}}{\omega^{2}}\right]
$$

In order to obtain this relation in the neighborhood of the parametric resonance frequency, we introduce

$\omega=\frac{1}{2}+\delta$

Here $\delta$ is a small quantity, whichs shows the variation of the natural frequency of the system about the resonance frequency.

We obtain the relation (3.12), in a more convenient form

$\delta=L a^{2} \pm \frac{1}{2} \sqrt{e^{2}-\frac{\gamma^{2}}{\omega^{2}}}$

where $L=\left[\frac{1}{16 \omega}-\frac{B \cos \delta}{64 \omega}-\frac{5 A}{16 \omega}\right]$

The eq.(3.14), is the relation between the amplitude of the stationary oscillations and frequency of the system. Equation (3.14), can be written in the form:

$\left(\delta-L a^{2}\right)^{2}=\frac{1}{4}\left(e^{2}-\frac{\gamma^{2}}{\omega^{2}}\right)$.

A schematic representation of behaviors of the relation (3.14) in the range of the parameter $\gamma$ for different values of ' $\mathrm{e}$ ' for e $=0.1,0.2,0.3,0.4$ and 0.5 , at $\omega=0.5$ represented by Fig.1 to Fig.10, for each values of 'e' there are three branches of $a=a(\delta)$, which are simulated using the software MATLAB 6.1 version. We observe that the branch 1 and 2 meets at and around ' $\mathrm{O}$ ' then the value of ' $\mathrm{a}$ ' are found imaginary corresponding to a value $\delta$. We also observe that as the value 'e' increases, the extraordinary change in the values of amplitude are also being observed,which are shown in the figures. The behaviors of the system are also investigated by simulating in three dimensional case taking into account the same values of parameters using MATLAB 6.1 version software, which exibits almost the same behavior as it was in the case of two dimensional simulation of the system.

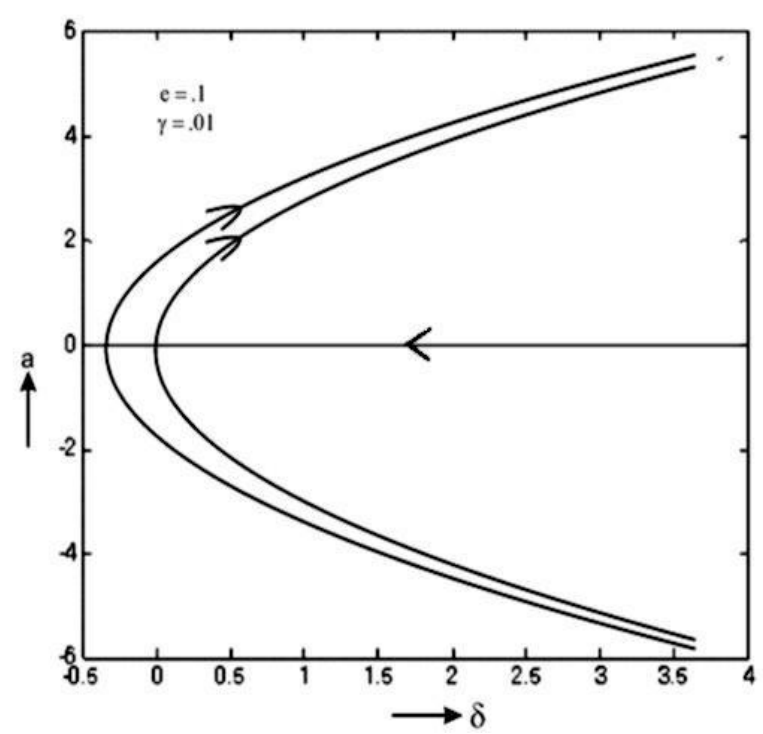

Fig.1: Behavior of the system under parametric resonance 


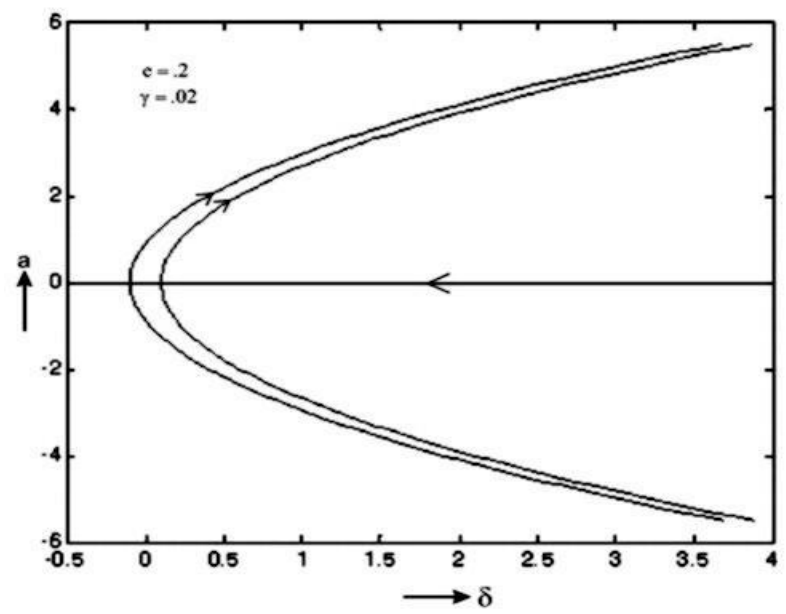

Fig. 2: Behavior of the system under parametric resonance

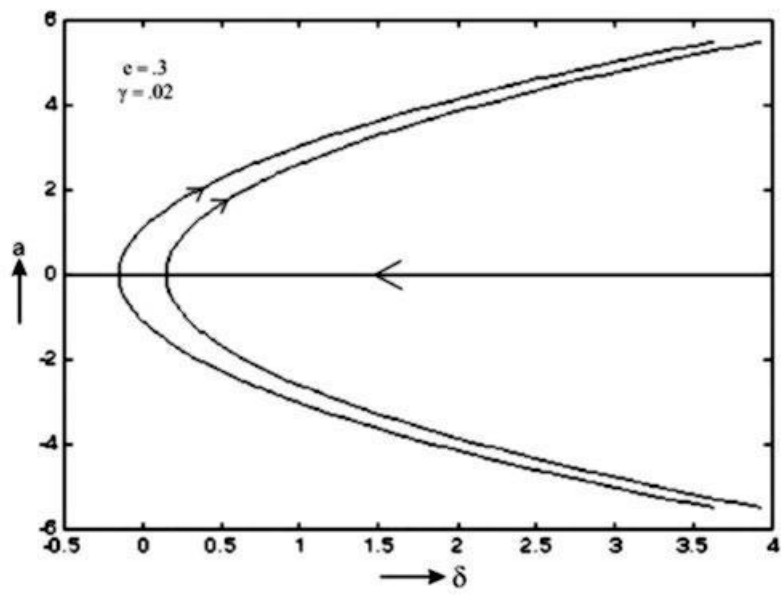

Fig. 3: Behavior of the system under parametric resonance

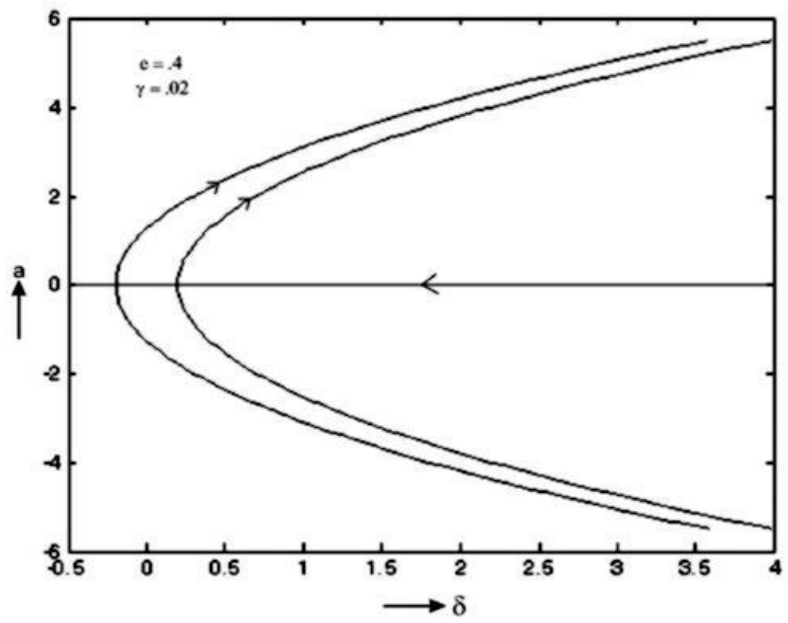

Fig. 4: Behavior of the system under parametric resonance 


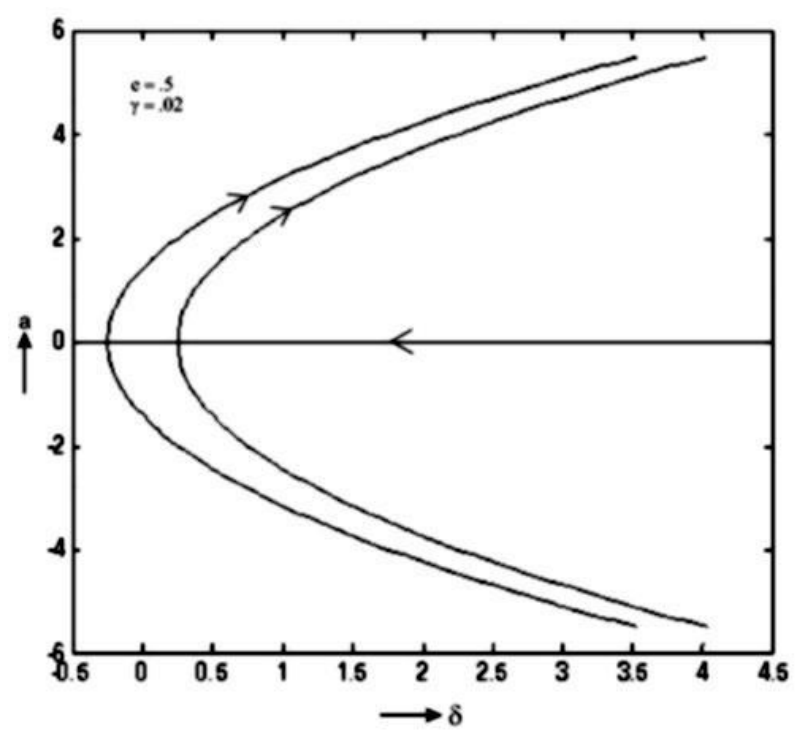

Fig. 5: Behavior of the system under parametric resonance

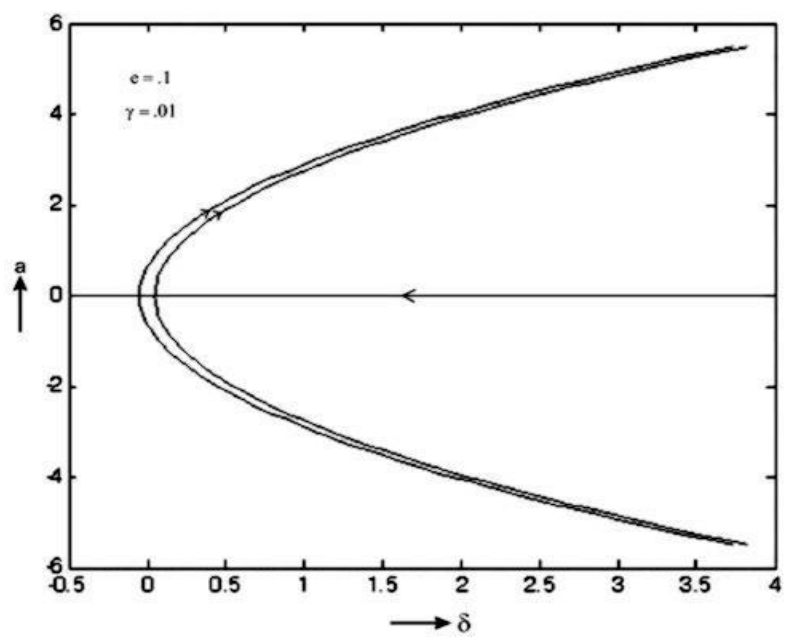

Fig. 6: Behavior of the system under parametric resonance

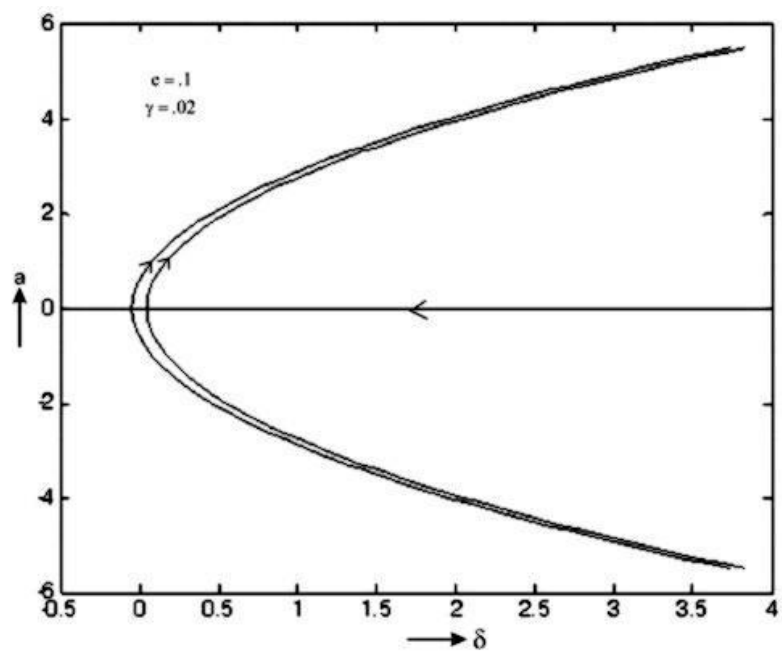

Fig. 7: Behavior of the system under parametric resonance 


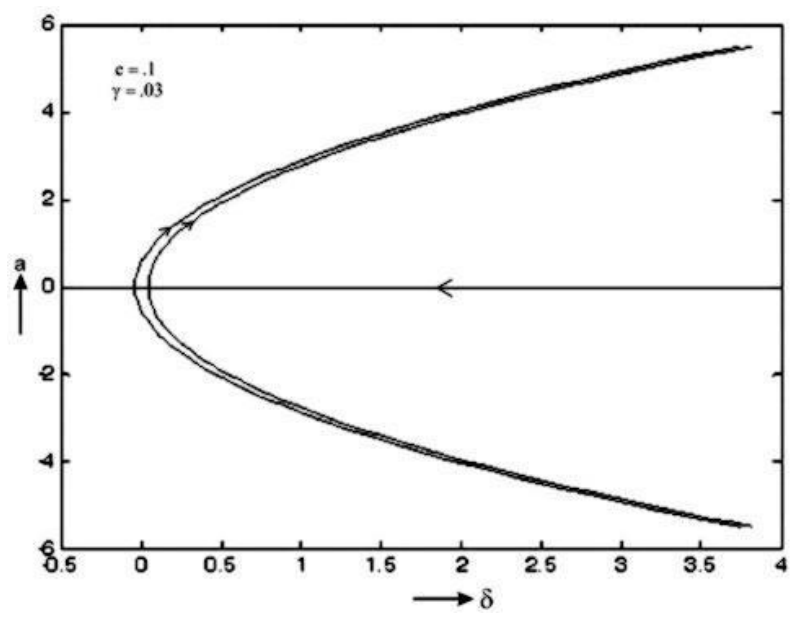

Fig. 8: Behavior of the system under parametric resonance

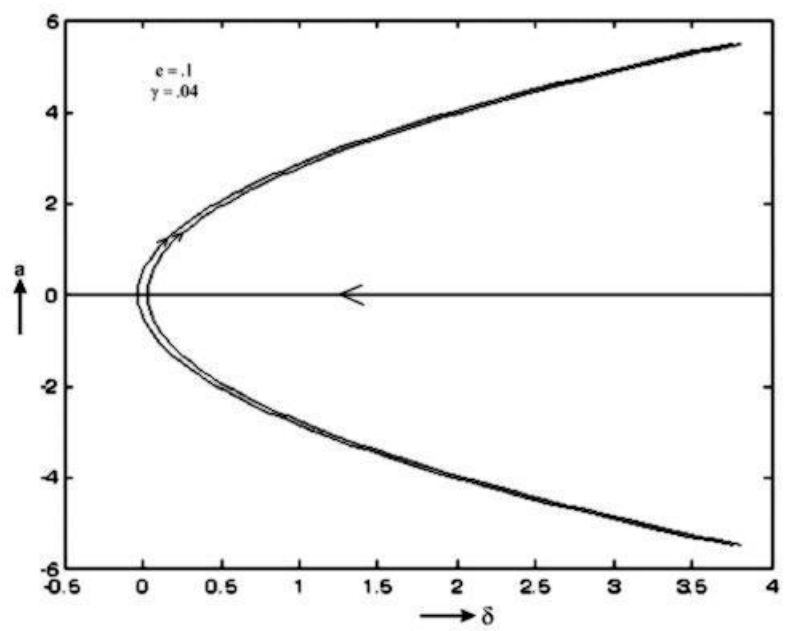

Fig. 9: Behavior of the system under parametric resonance

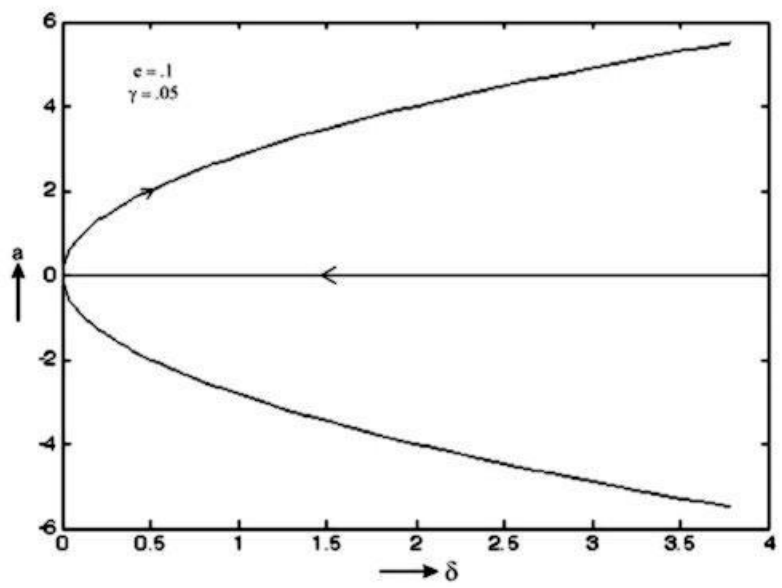

Fig. 10: Behavior of the system under parametric resonance 
We shall determine the relation that must exist between the parameter of the system for the effects under consideration to occur.The limit of this range are determined by the consideration $\frac{d a}{d \delta}=\infty$.

Proceeding with the relation (3.15), differentiating with respect to $\delta$, we obtain:

$\frac{d a}{d \delta}=\frac{\left(2 \delta-L a^{2}\right)}{\left(4 \delta L a-L^{2} 4 a^{3}\right)}$ which gives us

$\delta=L a^{2}$

We have also drawn the 3-D plots of the function using MATLAB 6.1.

$f(a, \delta)=4 \delta^{2} \omega^{2}+4 L^{2} \omega^{2} a^{4}-8 \delta L a^{2} \omega^{2}+\gamma^{2}-e^{2} \omega^{2}$

For the same values of parameter of 'e' and $\gamma$ the parametric resonance curve are plotted. The analysis regarding behavior of resonance curves is the same as in the case of two dimensional simulations which are also confirmed 3-D simulation given below from Fig.11 to Fig.20.

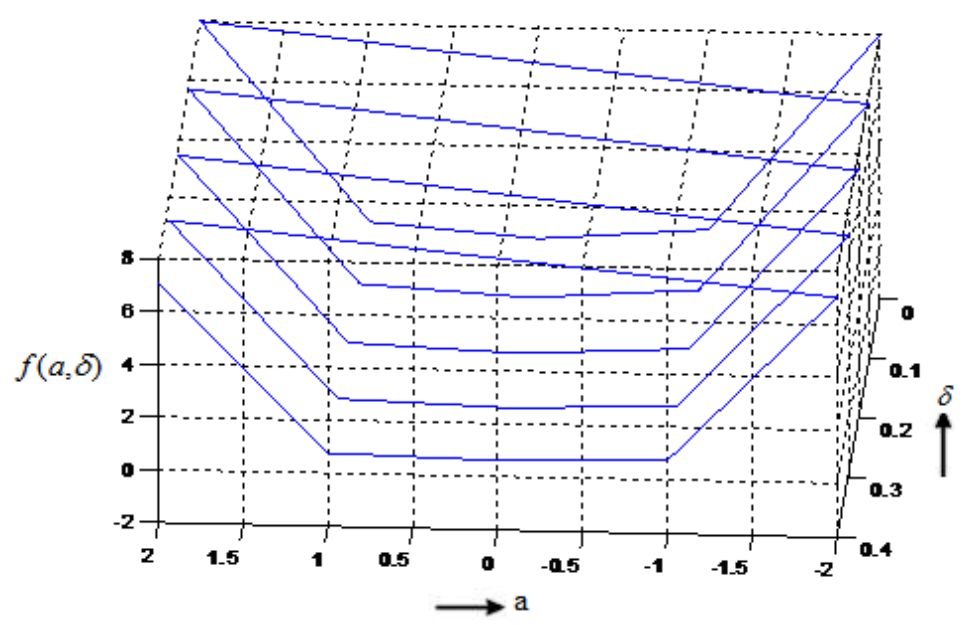

Fig. 11: Behavior of the system under parametric resonance at $\mathrm{e}=0.1$ and $\gamma=0.02$

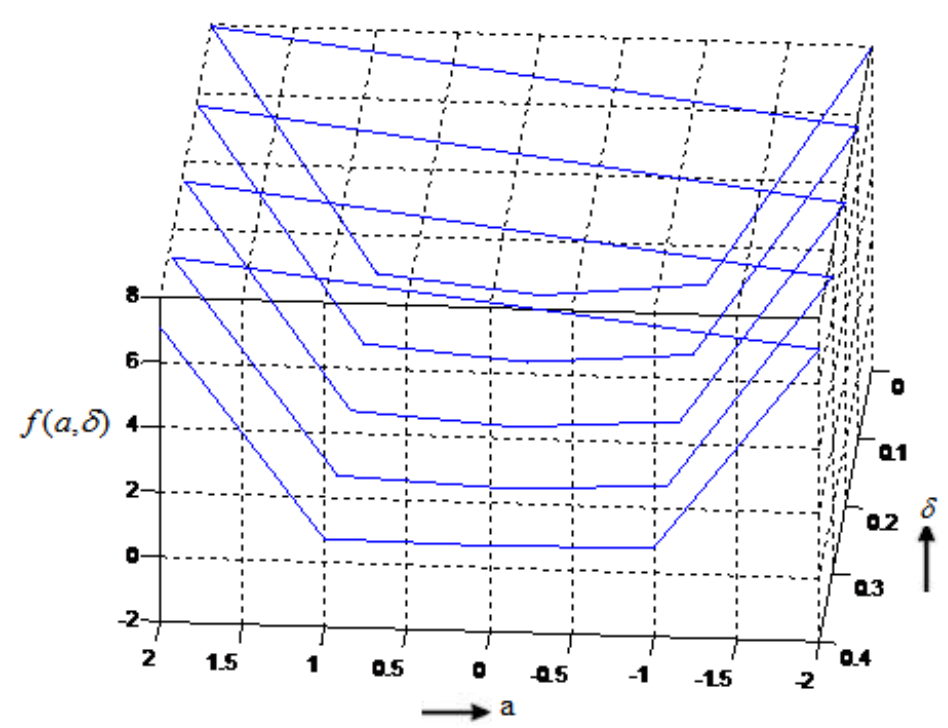

Fig.12: Behavior of the system under parametric resonance at $\mathrm{e}=0.2$ and $\gamma=0.02$ 


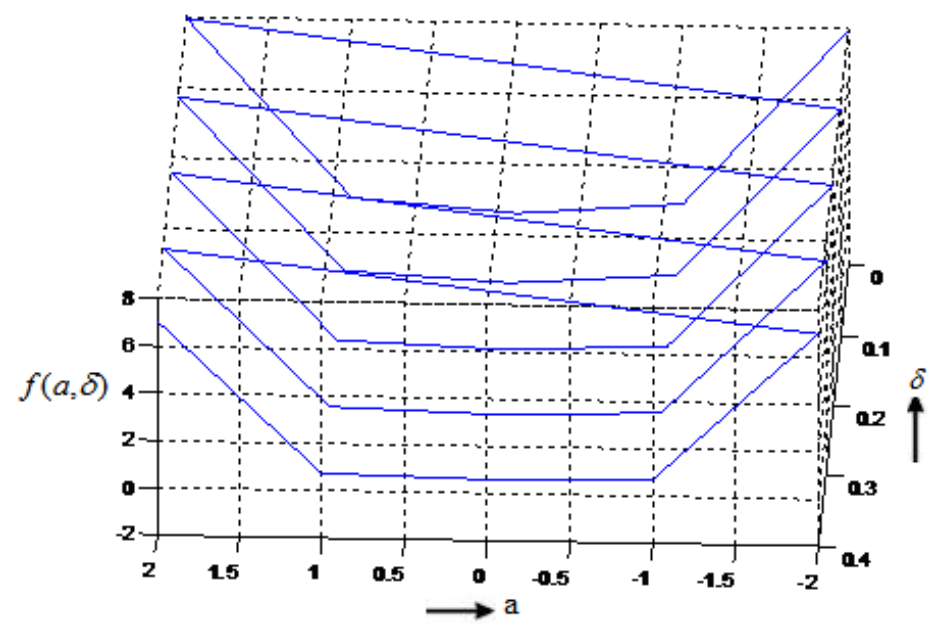

Fig. 13: Behavior of the system under parametric resonance at $\mathrm{e}=0.3$ and $\gamma=0.02$

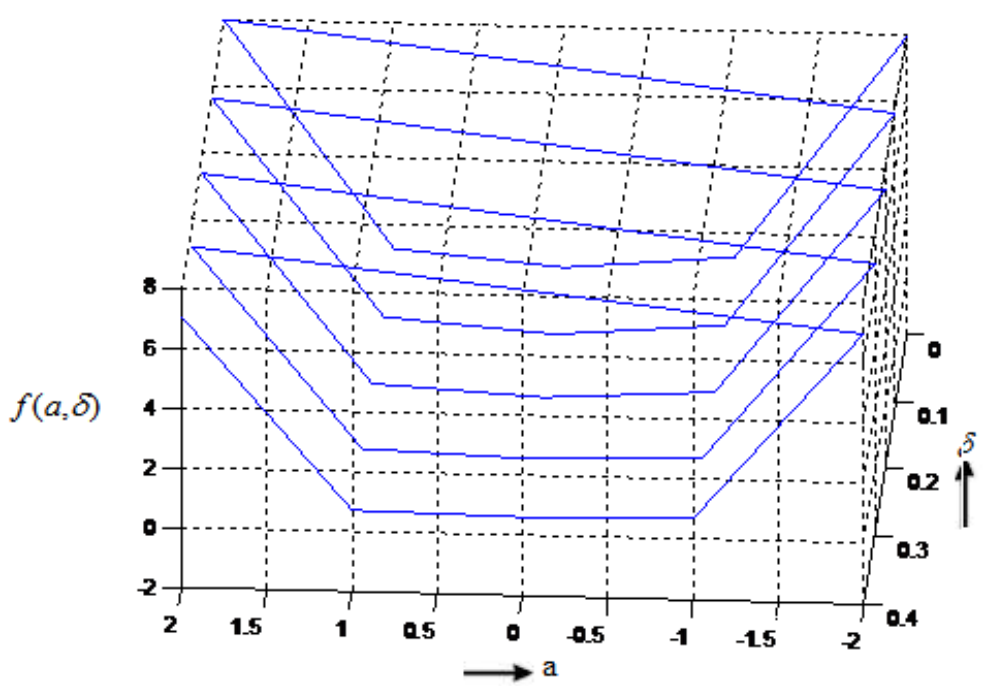

Fig. 14: Behavior of the system under parametric resonance at $\mathrm{e}=0.4$ and $\gamma=0.02$

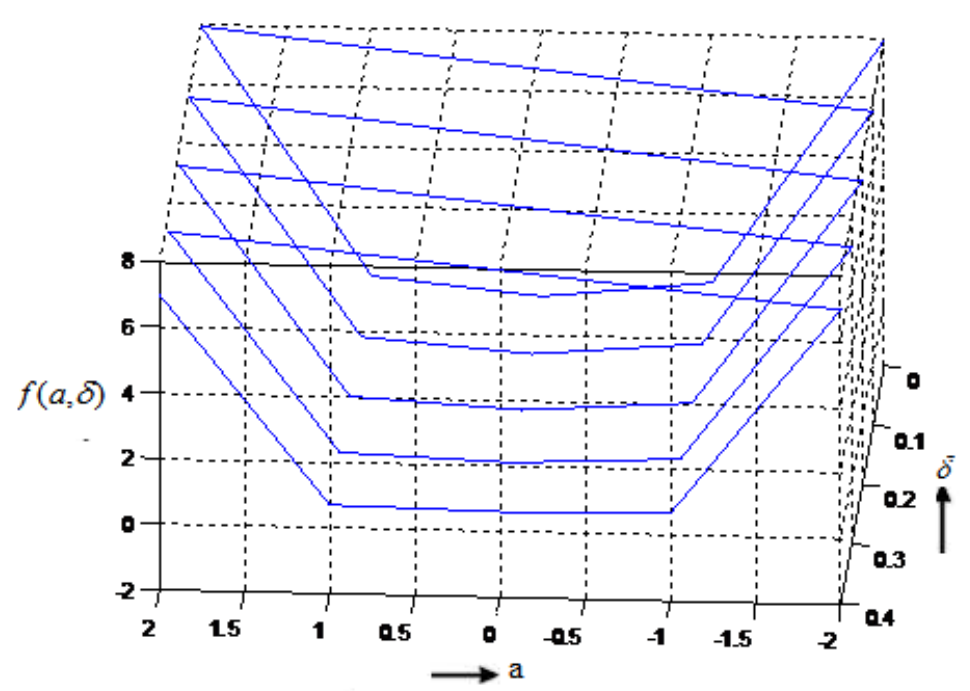

Fig. 15: Behavior of the system under parametric resonance at $\mathrm{e}=0.5$ and $\gamma=0.02$ 


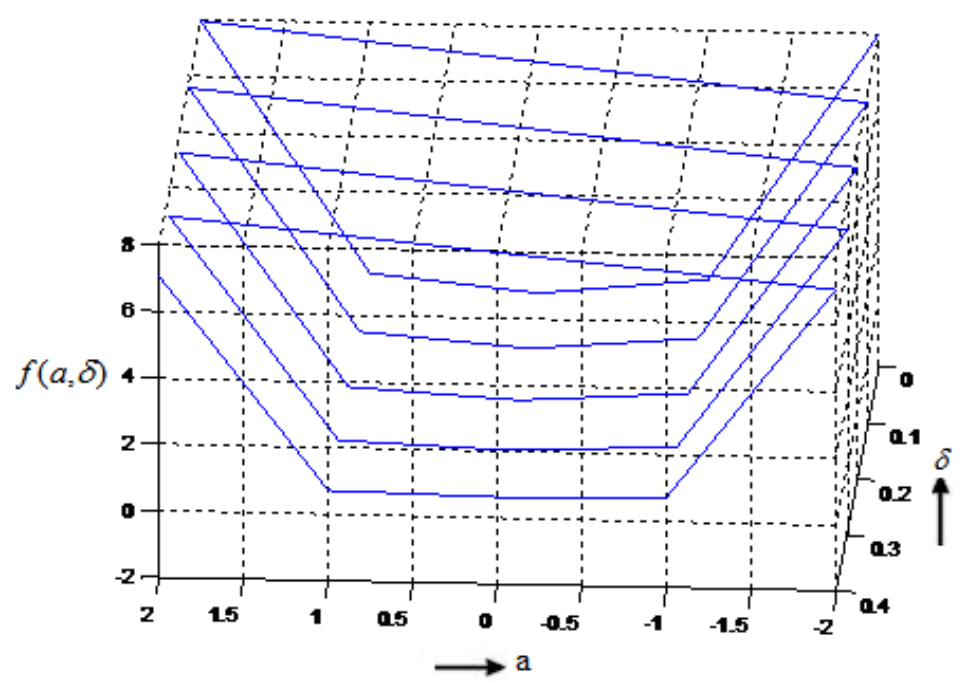

Fig. 16: Behavior of the system under parametric resonance at $\mathrm{e}=0.1$ and $\gamma=0.01$

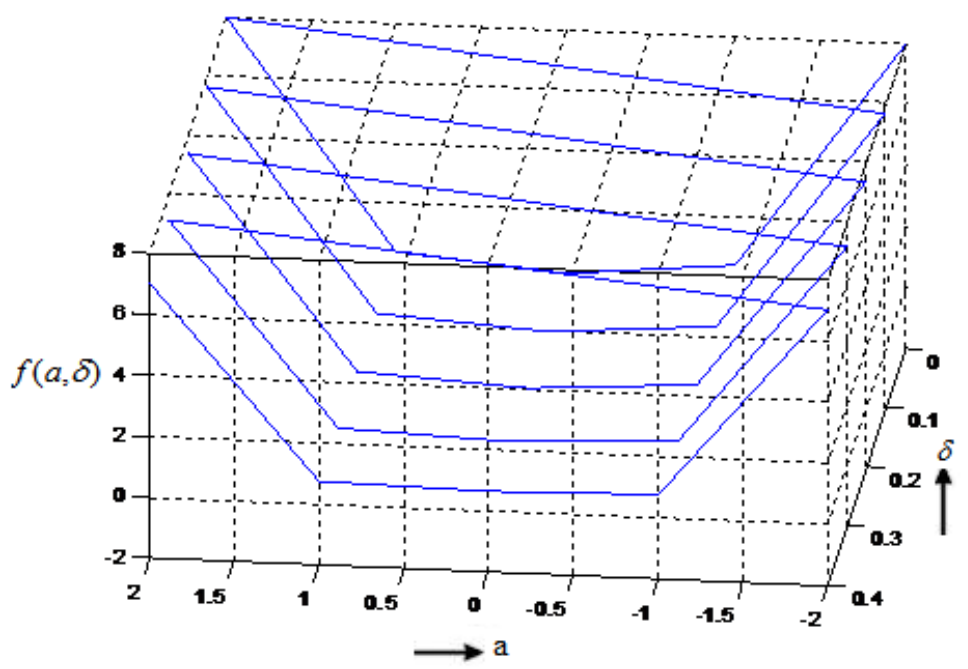

Fig. 17: Behavior of the system under parametric resonance at $\mathrm{e}=0.1$ and $\gamma=0.02$

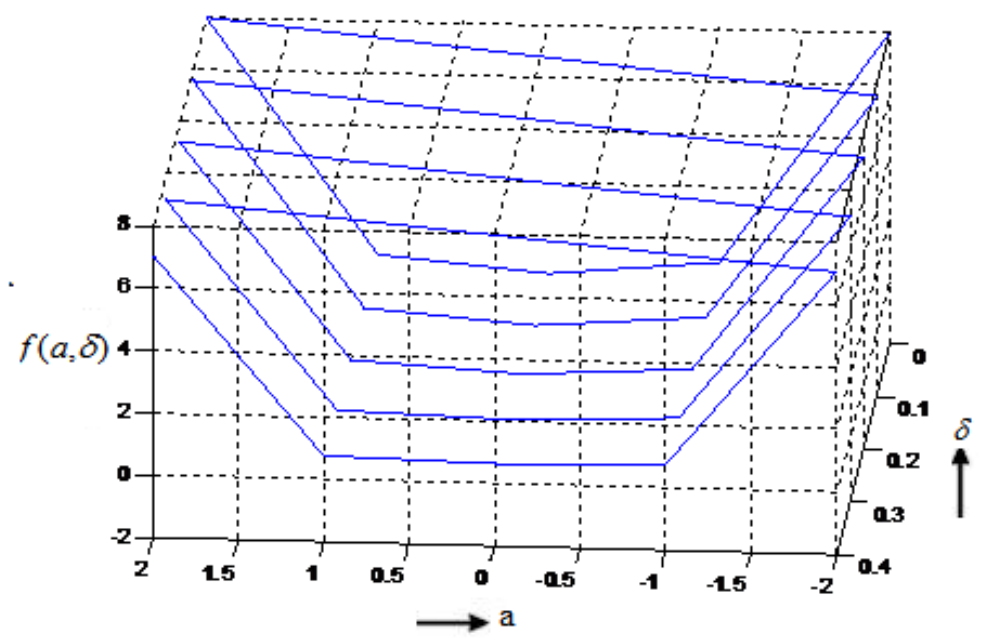

Fig.18: Behavior of the system under parametric resonance at $\mathrm{e}=0.1$ and $\gamma=0.03$ 


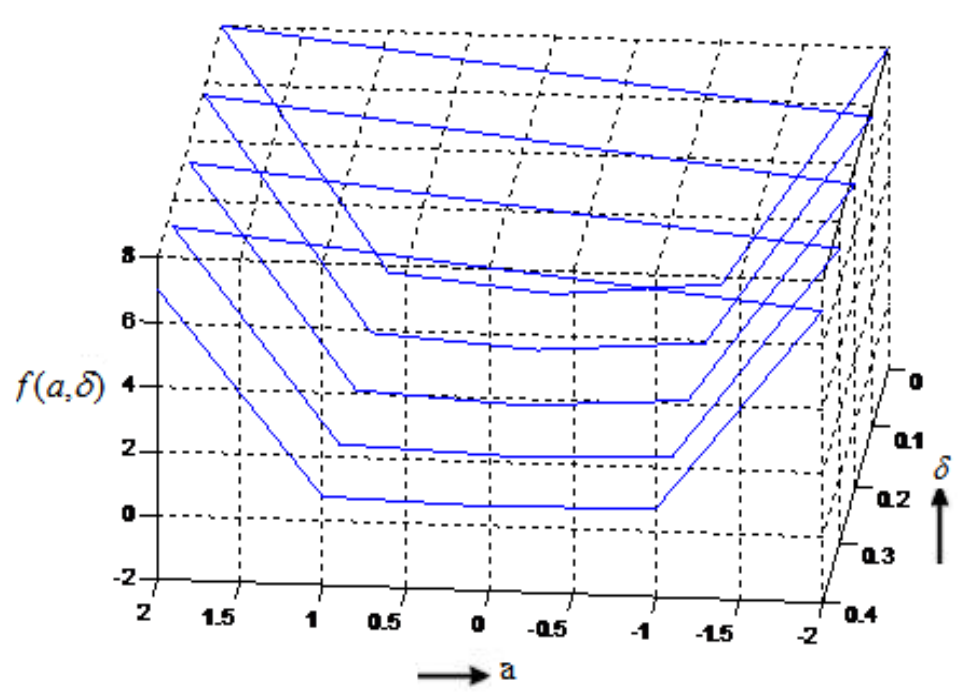

Fig. 19: Behavior of the system under parametric resonance at $\mathrm{e}=0.1$ and $\gamma=0.04$

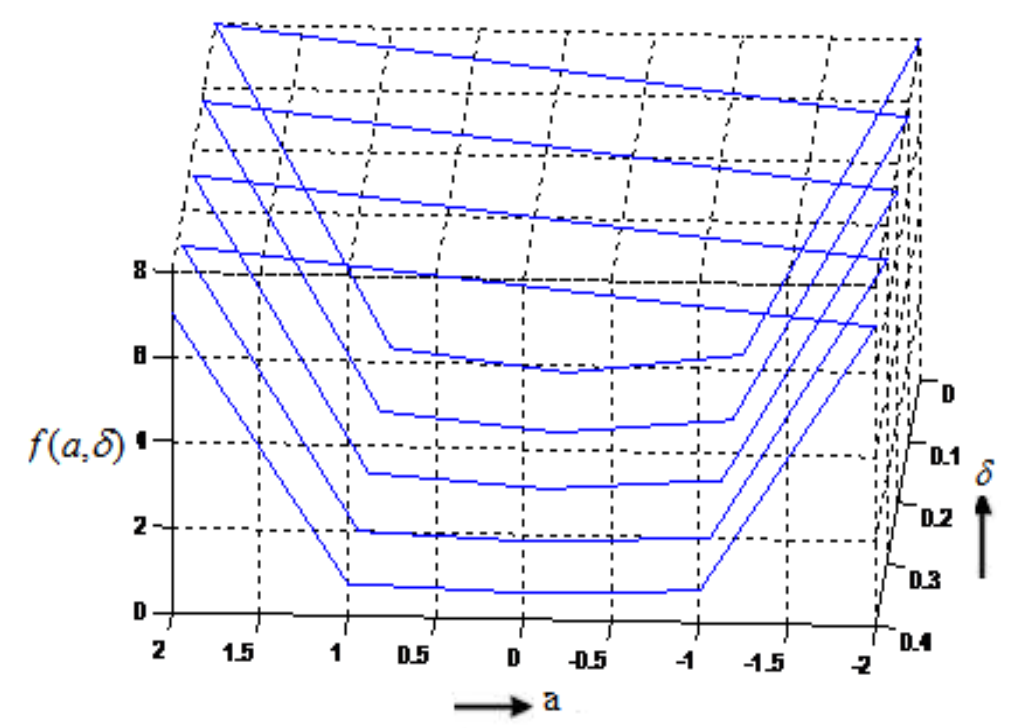

Fig. 20: Behavior of the system under parametric resonance at $\mathrm{e}=0.1$ and $\gamma=0.05$

\section{Discussion and conclusion}

The non-linear parametric resonance oscillations of dumbell satellite in elliptical orbit in the central gravitational field of Earth under the combined influence of the Earth magnetic field, Oblateness of the Earth and some external periodic forces of general nature has been discussed. Non-linear oscillations of dumbell satellites about the equilibrium position in the neighbourhood of the parametric resonance $\omega=\frac{1}{2}$, has been studied, exploiting the well known asymptotic method due to Bogoliubov,Krilov and metropolosky method,considering 'e' to be a small parameter. The stability of the system has been investigated using Poincare method.The effect under study that there is the break and jump in the amplitude of oscillations near the parametric resonance frequency $\omega=\frac{1}{2}$ are possible only for the small values of e and $\gamma$ taking into account of the magnetic field and oblateness of the Earth parameter. We arrive at the conclusion that the various perturbing force though small affects attitude of dumbell satellite in elliptical orbit near parametric resonance. 


\section{References}

[1] V.V. Beletsky, About the relative motion of two connected bodies in orbit, Kosmichekiya Isseldovania, vol-7 no. 06 (1969),827-840, In Russian.

[2] V.V. Beletsky, E.M. Levin, Dynamics of space tether systems, Advances of the Astronomical Sciences, vol.83 (1993), $267-322$.

[3] V.V. Beletsky, E.T. Novikova, About the relative motion of two connected bodies, Kosmichekiya Isseldovania, vol.7 no.03 (1969), 377-384, In Russian.

[4] Alessandria Celletti, Vladislov V. Sidorenko, Some properties of dumbbell satellite altitude dynamics, Celest Mech.\& Dyn. Astro. (2008), 105-126.

[5] S.K. Das, P.K. Bhattacharya, R.B. Singh, Effects of magnetic force on the motion of a system of two cable connected satellites in orbit, Proc. Nat. Acad. Sci. India (1976), 287-299.

[6] M. Krupa, A. Kuha, W.Poth, M. Schagrl, A. Stiensl, W. Steiner, H. Treger, G. Wiedermann, Tethered satellite systems: A new concept of space flight, European Journal of Mechanics. A Solids 19 - Special Issue (2000),S145-S164.

[7] M. Krupa, W. Poth, M. Sxhagerl, A. Stendl, W. Steiner, H. Treger, G. Wiedermann, Modelling, dynamics and central of tethered satellite systems, Non Linear Dynamics (2006), 73-96.

[8] A.M. Liapunov, The general problem of stability of motion, Sobrania Sachimediviya, vol. 2, A.N.Mosckow,In Russian.

[9] A.K. Mishra, V.J. Modi, Department and retrieval of shuttle supported tethered satellites, Journal of Guidance and Central, vol. 5, No. 3 (1982), 278- 285 .

[10] A. Narayan, R.B. Singh, Non-linear non-reasonance oscillation of interconnected satellites system under the solar pressure about the position of equilibrium for small eccentricity, Proc. Nat. Acad. Sci., India (1987), 427-437.

[11] A. Narayan, R.B. Singh, Nonlinear main resonance oscillation of the system of two interconnected satellites under the influence of solar pressure about stable equilibrium for small eccentricity, Proc. Nat.. Acad. Sci., India (1990), 307-315.

[12] A. Narayan, R.B. Singh, Non-linear parametric resonance oscillation of the system of two interconnected satellites under the influence of solar pressure about the stable position of equiliberiumfor small eccentricity ,Proc. Nat.. Acad. Sci., India (1992), vol.62 part II .

[13] Vladislov V.Sidorenko,Alessandra Celletti,A spring mass model of tethered satellites system properties of planar periodic motions ,celestial Mechanics Dynamical Astronomy 107.(2010).209-231.

[14] Ayub Khan,Neeti Goel,Chaotic motion in problem of dumbell satellite,International journal comtemp.Maths sciences vol.6 (2011)299-307.

[15] V. Nechvile, Surune Nouvelle formed equations diffierentielles due problem restraint elliptique comptc rendus, Acad. Paris, Compt. Rend. (1926), 182- 3100.

[16] S. Sharma, A. Narayan, Non-linear oscillation of inter connected satellite system under the combined influence of the solar radiation pressure and dissipative forces general nature, Bull. Astrtonomical Soc. India, 29 (2001).

[17] S. Sharma, A. Narayan, Effect of solar radiation pressure on the motion and stability of inter connected satellites system in orbit, Indian J. Pure Appl. Math., 33, No. 5 (2002), 609-623.

[18] R.B. Singh, Three dimensional motion of two-connected bodies in the cen- tral gravitational field of force, In Problem of Guided Motion in Mechanics, Russian Collection (1971), 210-215.

[19] R.B. Singh, Three dimensional motion of a system of two cable connected satellites in orbit, Astronautica Acta, 19 (1973), 301-308,

[20] B.M. Singh, A. Narayan, R.B. Singh, Non-linear parametric resonance oscillation of the system of two interconnected satellites orbiting around an oblate Earth, Proc. Nat. Acad. Sci., India, 67A (1997), 45-55.

[21] B.M. Singh, A. Narayan, R.B. Singh, Non-linear effects in the motion and stability of an inter connected satellites system orbiting around an oblate Earth, Proc. Nat. Acad. Sci., India, 71 (2001), 225-235.

[22] V.A.Sarychev and S.A.Mirer,Relative equilibria of a satellites subjected to gravitational and arodynamics torque's ,Celestial Mechanics\& Dynamical Astronomy 76 (2000).55- 68.

[23] V.A.Sarychev and S.A.Mirer,A.A.Degtyarev,E.K.Durate,Investigation equilibria of a satellite subjected to gravitational and arodynamic torque's,Celestial Mechanics\& Dynamical Astronomy (2007),267-287.

[24] J.F.Palacian, Dynamics of a satellites orbiting a planet with an inhomogeneous gravitational field ,Celestial Mechanics \& Dynamical Astronomy (2007),219-249.

[25] A.P.Markeev,B.S.Bardin,On the stability of planar oscillations and rotation of a satellites in circular orbit,Celestial Mechanics\& Dynamical Astronomy (2003),57-66.

[26] Cedric Langbort,Birfurcation of relative equilibria in the main problem of artificial satellite theory for a prolate orbit ,Celestial Mechanics \& Dynamical Astronomy (2002),369- 385.

[27] Bogoliubov N.N.,Mitropolosky Y.A., Asymtotic methods in the theory of non-linear oscillations,Hindustan publishing company Delhi 06 (1961).

[28] Landau L.D.,Lifschitz,E.M.,Mechanics Pergamon press oxford (1969).

[29] A. Narayan,S.Srivastav,S.Dewangan,Effects of earth magnetic field on the stability of cable connected satellites system in equitorial orbit ,Journal of AMSE modelling B (2004)45-60.

[30] A. Narayan,M.D.Pandey,Condition of free and constrained motion of cable connected satellites system in low altitude orbit ,(2010) IJPAM Bulgaria 107-127.

[31] A. Narayan,M.D.Pandey, Condition of non-linear stability of dumbell satellite in elliptical orbit .(2011) IJPAM Bulgaria 173-194.

[32] Rashmi Bhardwaj and K.B.Bhatnagar, Chaos in the nonlinear planar oscillation of a satellite in an elliptical orbit under the influence of third body torque.(1997) Indian J.Pure applied maths. 391-422.

[33] A.Narayan,M.D.Pandey and Amitesh Narayan, Some nonlinear resonance oscillations of dumbell satellite in elliptical orbit. (2012) IJPAM Bulgaria 931-944. 